Research Paper

\title{
Treatment of Relapsed/Refractory Hodgkin Lymphoma: Real-World Data from the Czech Republic and Slovakia
}

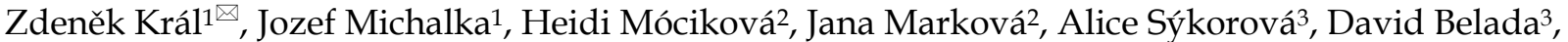 \\ Alexandra Jungová4 ${ }^{4}$ Samuel Vokurka ${ }^{4}$, Marie Lukášová5 , Vít Procházka ${ }^{5}$, Juraj Duraš ${ }^{6}$, Roman Hájek ${ }^{6}$, \\ Ladislav Dušek ${ }^{7}$, Ĺuboš Drgoňa ${ }^{8}$, Miriam Ladická8, Veronika Ballová9 ${ }^{9}$ Andrej Vranovský ${ }^{8}$
}

1. Department of Internal Medicine, Hematology and Oncology, University Hospital Brno and Faculty of Medicine, Masaryk University, Brno, Czech Republic

2. Department of Clinical Hematology, University Hospital Kralovske Vinohrady and Third Faculty of Medicine, Charles University, Prague, Czech Republic

3. 4th Department of Internal Medicine - Hematology, University Hospital Hradec Kralove, Czech Republic and Charles University in Prague, Faculty of Medicine in Hradec Kralove, Czech Republic

4. Department of Haemato-Oncology, University Hospital in Plzen, Plzen 304 60, Czech Republic

5. Department of Hemato-Oncology, Faculty of Medicine and Dentistry, Palacký University, Olomouc, Czech Republic

6. Department of Hemato-Oncology, Faculty of Medicine, University of Ostrava and University Hospital Ostrava, Ostrava, Czech Republic

7. Institute of Biostatistics and Analyses, Faculty of Medicine, Masaryk University, Czech Republic

8. Department of Oncohematology, Comenius University in Bratislava and National Cancer Institute, Bratislava, Slovakia

9. Department of Hematology/Oncology, Kantonsspital Baden, Baden, Switzerland

$\square$ Corresponding author: MUDr. Zdeněk Král, CSc., Department of Internal Medicine, Hematology and Oncology, University Hospital Brno and Faculty of Medicine, Masaryk University, Jihlavská 340/20, 62500 Brno, Czech Republic. Tel: +421 532 233 642; Fax: +421 532 233 603; Email: Kral.Zdenek@fnbrno.cz

(C) The author(s). This is an open access article distributed under the terms of the Creative Commons Attribution License (https://creativecommons.org/licenses/by/4.0/). See http://ivyspring.com/terms for full terms and conditions.

Received: 2018.08.17; Accepted: 2019.07.13; Published: 2019.08.28

\begin{abstract}
Introduction: Clinical trials have demonstrated the effectiveness of the CD30-targeted antibodydrug conjugate brentuximab vedotin (BV) for the treatment of relapsed/refractory Hodgkin lymphoma $(\mathrm{R} / \mathrm{R} \mathrm{HL})$. In this study, we report on outcomes with $\mathrm{BV}$ in a real-world setting using data collected in clinics in the Czech Republic and Slovakia.

Patients and Methods: Clinical and epidemiological data for patients with R/R HL who received treatment with BV at eight centers across the Czech Republic and Slovakia were examined. Data were amalgamated and analyzed retrospectively.

Results: Clinical data for 58 patients (median age: 30.5 years) with R/R HL who received BV during the course of their treatment were collected and analyzed. Patients had received a median of 3 prior treatment regimens and most $(91 \%)$ were treated with BV after relapse following autologous stem cell transplantation. Therapeutic responses after BV included 19 (33\%) complete responses (CRs) and $8(14 \%)$ partial responses. CRs occurred more frequently in patients who had received fewer prior treatment regimens. The 1-, 2-, and 3-year overall survival (OS) rates from initiation of BV were $78 \%, 62 \%$, and $41 \%$, respectively.

Conclusion: Response rates and OS in this analysis of BV in real-world settings in the Czech Republic and Slovakia were consistent with those reported for pivotal clinical trials and from previous studies outside the clinical trial setting. The results support the efficacy of BV for treatment of $R / R H L$ in real-life clinical practice.
\end{abstract}

Key words: antibody-drug conjugate; CD30; brentuximab vedotin; Hodgkin lymphoma; registries; stem cell transplantation

\section{Introduction}

Treatment for Hodgkin lymphoma (HL) achieves very high cure rates, with most patients $(>80 \%)$ achieving a cure and long-term survival.
However, approximately $20-40 \%$ of patients experience a relapse after front-line therapy or fail to respond to initial treatment, with approximately $50 \%$ 
of these patients being subsequently salvaged by high-dose chemotherapy followed by autologous stem cell transplantation (ASCT), which is the standard of care for most patients according to the European Society for Medical Oncology (ESMO) guidelines for the management of HL. ${ }^{1-5}$ In patients with failure after ASCT the outlook is poor, with a median survival of only 25 months. ${ }^{6}$

A number of factors that are predictive of outcome after ASCT have been identified, such as early ( $<12$ months) relapse after ASCT, disease refractory to front-line therapy, failure to achieve a response to the most recent salvage therapy, extranodal disease (stage IV) or B-symptoms at pre-ASCT relapse, prior use of two or more salvage therapies, bulky disease, poor performance status, and age $\geq 50$ years at relapse. Patients with one or more of these factors have worse outcomes after ASCT. ${ }^{7}$ Furthermore, some patients are not eligible for ASCT due to factors such as age, refractory disease, or poor performance status. For these patients, new treatment strategies are needed urgently.

Brentuximab vedotin (BV) is a CD30-targeting antibody-drug conjugate that was shown in a pivotal phase II trial to be an effective and well-tolerated treatment for patients with relapsed/refractory $(R / R)$ HL after ASCT with an overall objective response rate (ORR) of $75 \%$ and complete remission in $34 \%$ of patients. ${ }^{8}$ Recently published 5-year follow-up data from the trial showed that durable responses could be achieved even without further anticancer therapy, with 9 of the 34 patients (26\%) who achieved a complete response (CR) still in remission and potentially cured. ${ }^{9}$ Studies have also shown that BV is an effective option for patients with R/R HL who are ineligible for transplantation. ${ }^{10,11}$

While clinical trials are critical for establishing efficacy, collection of real-world data outside of the controlled trial setting is important to evaluate how interventions are applied and assess the effectiveness of new treatments in routine clinical practice. Inclusion criteria are often rather restrictive compared with the patient populations seen by physicians in daily practice.

There are limited real-world data related to treatment with BV, and where it is available, efficacy and safety are consistent with those seen in clinical trials. Five retrospective observational studies have collected data for more than 200 patients with R/R HL treated with BV in centers in Asia, France, Italy, and Turkey.12-16 Across the studies, ORRs were in the range of $40-73 \%$, and CRs were reported for $18-34 \%$ of patients. For the four studies that reported median progression-free survival (PFS), these ranged from 6.6 to 9.0 months. The most frequently observed adverse events across the studies included sensory neuropathy and neutropenia.

The present study investigates a population of patients who have received a median of 3 previous treatment regimens. These patients represent those who have relapsed and may then have a critical medical need, requiring a different management strategy to standard salvage therapy. We report the results of a retrospective, observational study with the objective of assessing the effectiveness and tolerability of $\mathrm{BV}$ for the treatment of R/R HL in a real-world setting, based on complementary data collected in a collaboration between institutes in the Czech Republic and Slovakia.

\section{Methods}

This retrospective, observational study examined data for patients with R/R HL at eight centers in the Czech Republic and Slovakia. In the Czech Republic, data came from a clinical registry of patients with R/R HL who started treatment with BV between May 2013 and November 2015. An identical data collection strategy was used in Slovakia for patients who started BV treatment between July 2012 and January 2016. All patients were treated within government-funded schemes, with no patients included from clinical trials or named patient programs. Patients were CD30-positive, as determined by immunohistochemical examination on entry biopsy, typically with positivity in the membrane and Golgi apparatus in Hodgkin's and Reed-Sternberg cells of classical HL.

The analysis included data for patients who received $B V$ for the treatment of $R / R$ HL following ASCT or who were ineligible for ASCT after failure of one previous salvage therapy. BV was initiated at the recommended dosing of $1.8 \mathrm{mg} / \mathrm{kg}$ every 3 weeks for up to a maximum of 16 cycles.

The data collected and examined included patient characteristics and demographics, prior treatment history, details of BV treatment, clinical responses to BV treatment, and survival outcomes. Clinical responses were evaluated according to the revised Cheson criteria (2007), ${ }^{17}$ with all patients assessed by computed tomography before initiating BV and after 4, 8, 12, and 16 cycles of treatment as applicable. Use of positron emission tomography (PET) was not reimbursed within the government-funded schemes and was not routinely available. Overall survival (OS) was determined both from the time of HL diagnosis and the initiation of $\mathrm{BV}$ treatment.

Primary data were expressed using absolute and relative frequencies for categorical variables, and median and range (5-95 percentiles) for continuous 
variables. Differences between patient groups were assessed using the non-parametric Fisher exact test, Mann-Whitney U test, or Kruskal-Wallis test.

The analyses employed logistic regression models to quantify the association between potential predictors and principal, binary-coded endpoints (i.e. relapse rate, response to the therapy) using both univariate and multivariate-adjusted approaches. The models used maximum likelihood estimation, directly comparing the likelihood L0 for the null model where all slope parameters are zero, with the likelihood L1 of the fitted model. Significance of regression coefficients was tested using the Wald test statistic, which is based on the asymptotic normality property of maximum likelihood estimates (tested on the basis of Chi-square distribution). Similarly, univariate and multivariate Cox proportional hazard regression models were applied to test the impact of potential predictors on OS as a time-to-event endpoint. Both univariate and multivariate-adjusted estimates of odds ratio (OR) and hazard ratio (HR) were determined with 95\% confidence intervals. Standard Kaplan-Meier curves were used to display OS profiles stratified according to clinical stage. Log-rank testing was used to assess the statistical significance of differences across the strata.

The study was conducted according to the principles expressed in the Declaration of Helsinki. Informed consent from the participants was not collected as all data were analyzed anonymously.

\section{Results}

\section{Patient characteristics and treatment history}

A total of 58 patients with R/R HL who had received treatment with $\mathrm{BV}$ were identified in the institutional databases and included in the retrospective analysis. Patient and disease characteristics, as well as the treatment history for the 58 patients, are summarized in Table 1 . The median age of patients at diagnosis was 30.5 years, and the median follow-up from diagnosis was 4.3 years from diagnosis and 1.4 years from BV initiation.

Patients had received a median of 3 treatment regimens prior to treatment with $\mathrm{BV}$. The majority of patients $(86 \%)$ had previously received doxorubicin/bleomycin/vinblastine/dacarbazine

(ABVD) at some point in the course of their treatment. Other frequently used chemotherapy regimens included bleomycin/etoposide/doxorubicin/cyclophosphamide/vincristine/procarbazine/pred-

nisolone (BEACOPP), dexamethasone/high-dose cytarabine/cisplatin (DHAP) and ifosfamide/ carboplatin/etoposide (ICE). The proportion of patients who had received radiotherapy was $69 \%$.
Most patients (91\%) had received at least one ASCT and $12 \%$ had had an allogeneic stem cell transplant (allo-SCT). Of the patients receiving prior ASCT, 7 patients underwent 2 transplants prior to $\mathrm{BV}$ administration, three of which were tandem-ASCTs with an inter-transplant interval of 5-7 months. Based on the activity of the disease, patients in this study were divided into two groups prior to initiating BV 52 patients $(90 \%)$ were in relapse with progressive disease (PD) and 6 patients (10\%) had partial stabilization (i.e. they had either partial response [PR] or stable disease [SD]). Patients with PR/SD receiving BV treatment were those whose prior therapy did not lead to CR, despite 5 of these 6 patients having undergone ASCT, two of which were tandem-ASCTs.

Table 1: Patient characteristics and treatment history

\begin{tabular}{|c|c|c|c|}
\hline \multirow{2}{*}{\multicolumn{2}{|c|}{ 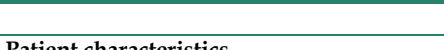 }} & $\mathbf{N}(\%)$ & Median (5-95 percentiles) \\
\hline & \multicolumn{3}{|c|}{ Patient characteristics } \\
\hline \multicolumn{2}{|l|}{$\begin{array}{l}\text { Length of follow-up } \\
\text { (years) }\end{array}$} & 4.3 & $(1.2-16.0)$ \\
\hline \multicolumn{2}{|l|}{$\begin{array}{l}\text { Age at time of } \\
\text { diagnosis }\end{array}$} & 30.5 & $(20.0-53.0)$ \\
\hline \multirow[t]{2}{*}{ Sex } & Men & 33 & (57) \\
\hline & Women & 25 & (43) \\
\hline \multirow[t]{3}{*}{ HL stage } & $\mathrm{I}+\mathrm{II}$ & 23 & (40) \\
\hline & III & 19 & (33) \\
\hline & IV & 16 & (28) \\
\hline \multirow[t]{2}{*}{ Indication for BV } & $\begin{array}{l}\text { Relapse after } \\
\text { autologous } \\
\text { transplantation }\end{array}$ & 53 & (91) \\
\hline & $\begin{array}{l}R / R \text {, unsuitable } \\
\text { for ASCT }\end{array}$ & 5 & (9) \\
\hline \multicolumn{4}{|l|}{ Pretreatment } \\
\hline \multicolumn{2}{|l|}{ Number of regimens } & 3.0 & $(2.0-9.0)$ \\
\hline \multirow[t]{8}{*}{ Previous regimens $\mathrm{s}^{\mathrm{a}}$} & ABVD & 50 & (86) \\
\hline & BEACOPP & 21 & (36) \\
\hline & COPP & 15 & (26) \\
\hline & DHAP & 26 & (45) \\
\hline & Gemcitabine & 9 & (16) \\
\hline & ICE & 26 & (45) \\
\hline & IGEV & 9 & (16) \\
\hline & Other & 29 & (50) \\
\hline \multirow[t]{2}{*}{ Radiotherapy } & No & 18 & (31) \\
\hline & Yes & 40 & (69) \\
\hline \multirow[t]{3}{*}{ ASCT } & No & 5 & (9) \\
\hline & Yes -1 transplant & 46 & (79) \\
\hline & Yes - 2 transplants & 7 & (12) \\
\hline \multirow[t]{2}{*}{ Allo-SCT } & No & 51 & (88) \\
\hline & Yes & 7 & (12) \\
\hline \multirow[t]{2}{*}{ Response before BV } & PD/relapse & 52 & (90) \\
\hline & $\mathrm{PR} / \mathrm{SD}$ & 6 & (10) \\
\hline
\end{tabular}

aTreated with regimen at any stage in treatment history; only regimens assigned to at least five patients are listed. ABVD: doxorubicin, bleomycin, vinblastine and dacarbazine; allo-SCT: allogenic stem cell transplantation; ASCT: autologous stem cell transplantation; BEACOPP: bleomycin, etoposide, doxorubicin,

cyclophosphamide, vincristine, procarbazine and prednisolone; BV: brentuximab vedotin; COPP: cyclophosphamide, vincristine, procarbazine and prednisolone;

DHAP: dexamethasone, high-dose cytarabine and cisplatin; HL: Hodgkin lymphoma; ICE: ifosfamide, carboplatin and etoposide; IGEV: ifosfamide, gemcitabine and vinorelbine; PD: progressive disease; PR: partial response; R/R: relapsed/refractory; SD, stable disease.

\section{Safety}

All patients received at least 3 doses of $B V$ with a median 7.5 cycles (range, 3-16 cycles). Only 1 patient $(2 \%)$ required a reduction in the dose of $\mathrm{BV}$ to 1.2 
$\mathrm{mg} / \mathrm{kg}$ due to neutropenia (Grade 3/4), while dosing delays were instituted for $8(14 \%)$ of the patients. Toxic effects of BV which lead to a prolongation of the dose interval included 2 cases of leukopenia (Grade $3 / 4), 1$ case of urinary tract infection, 2 cases of acute bronchitis, 1 case of odontogenic infection and 1 case of increase in pancreatic amylase. In our retrospective study, the most common toxic manifestation of BV was neurological toxicity, which was observed in 21 patients $(36.2 \%)$. Of these, 12 patients presented with peripheral sensory neuropathy (Grade 1/2) only, 3 patients with peripheral motor neuropathy (Grade 1/2) only, and 6 patients with simultaneous occurrence of sensory and motor neuropathy (Grade $1 / 2)$.

\section{Therapeutic responses to brentuximab vedotin}

Patients received a median of 7.5 cycles of BV treatment. Of the 58 patients, $19(33 \%)$ had a CR following BV treatment, with $8(14 \%)$ having a PR and 31 (53\%) having PD (Table 2). Discontinuation of BV was due to disease progression, negotiations with health insurance companies, or receipt of allo-SCT. Following treatment failure with BV, 6 patients received nivolumab, with 3 patients subsequently achieving CR. Of the remaining 3 patients, 2 progressed and 1 died of septic shock while on nivolumab therapy.

Table 2: Treatment and outcomes after brentuximab vedotin treatment

\begin{tabular}{|c|c|c|c|}
\hline & & $\mathrm{N}(\%)$ & Median (5-95 percentiles) \\
\hline \multicolumn{4}{|l|}{ BV treatment } \\
\hline Number of BV cycles & & 7.5 & $(3.0-16.0)$ \\
\hline \multirow[t]{3}{*}{ Number of BV cycles } & $2-5$ & 20 & (35) \\
\hline & $6-10$ & 29 & (50) \\
\hline & $11-16$ & 9 & (16) \\
\hline \multirow[t]{2}{*}{ Dose reduction } & No & 57 & (98) \\
\hline & Yes & 1 & (2) \\
\hline \multirow[t]{2}{*}{ Interval change ${ }^{a}$} & No & 16 & (67) \\
\hline & Yes & 8 & (33) \\
\hline \multirow[t]{5}{*}{ Response during treatment } & $\mathrm{CR}$ & 8 & (14) \\
\hline & PR & 26 & (45) \\
\hline & SD & 7 & (12) \\
\hline & PD & 12 & (21) \\
\hline & Not available & 5 & (9) \\
\hline \multicolumn{4}{|l|}{ Outcome } \\
\hline \multirow[t]{3}{*}{ Response on BV } & CR & 19 & (33) \\
\hline & PR & 8 & (14) \\
\hline & PD & 31 & (53) \\
\hline \multirow[t]{2}{*}{ Relapseb $^{b}$} & No & 21 & (78) \\
\hline & Yes & 6 & (22) \\
\hline \multirow[t]{2}{*}{ Death } & No & 38 & (66) \\
\hline & Yes & 20 & (35) \\
\hline \multicolumn{4}{|c|}{$\begin{array}{l}\text { Data are presented as } \mathrm{N}(\%) \text { or median (5-95 percentiles). aData are not available for } \\
34 \text { patients. bRelapse occurrence was assessed only in patients with a CR or PR on } \\
\text { BV treatment. }\end{array}$} \\
\hline $\begin{array}{l}\mathrm{BV} \text { : brentuximab vedotin; } \mathrm{Cl} \\
\text { partial response; } \mathrm{SD} \text { : stable }\end{array}$ & complete resp & nse; $\mathrm{PD}$ & : progressive disease; PR: \\
\hline
\end{tabular}

Of the 58 patients treated with $\mathrm{BV}$, the duration of the therapeutic response was evaluated in 34 patients, with the median being 5 months. Of the remaining patients, 13 patients achieving a response to $\mathrm{BV}$ were directed to allo-SCT, and the remaining 11 patients are alive with no signs of relapse/ progression of HL.

Patients who achieved a CR after BV subsequently received more cycles of treatment (median 8 cycles) as they did not relapse during BV treatment. Patients with an inadequate response to BV received fewer cycles (PR, median 5.5 cycles; PD, median 6 cycles) mostly due to the poor response $(P<0.001)$. BV treatment was discontinued early (patients received $<8$ cycles of BV) in a total of 29 patients. Of these, 19 patients discontinued treatment due to insufficient response to BV. Six patients discontinued BV treatment early due to redirection to allo-SCT, of which 3 patients were in PR following 3, 5 and 6 cycles of BV, and 3 patients were in CR following 6, 3 and 6 cycles. The number of BV cycles was also influenced by negotiations with health insurance companies, which lead to the decision to discontinue BV treatment early in 2 patients who achieved CR. No patients discontinued treatment early due to toxicity or drug availability issues, and the remaining 2 patients discontinued treatment early due to unknown reasons.

Patients receiving fewer previous treatment regimens were more likely to achieve a CR. Univariate and multivariate logistic regression models confirmed these associations, and that none of the other factors tested were significantly correlated with the likelihood of achieving a CR (data in Supplementary Table S1).

No factor was found to be significantly associated with the risk of relapse in univariate or multivariate logistic regression models (Supplementary Table S2). Overall, 14 patients underwent allo-SCT after BV treatment. CR was achieved following allo-SCT in all patients who received the transplant in CR (6 patients) or PR (3 patients). Of the remaining 5 patients receiving allo-SCT with PD, CR was achieved in 2 cases.

\section{Progression-free survival}

Figure 1 shows the PFS for the 58 patients from the start of BV therapy. The PFS rates at 1 and 2 years were $63.2 \%$ and $45.2 \%$, respectively. The median PFS was $1.38(0.56-2.21)$ years.

\section{Overall survival}

At 1 and 3 years after initiation of BV, OS was $78 \%$ and $41 \%$, respectively (Figure $2 \mathrm{~A}$ ). The OS rate at 1 year after BV initiation was $96 \%$ for patients with Stage I or II disease at diagnosis, compared with $78 \%$ for Stage III disease and $56 \%$ for Stage IV disease (Figure 2B). In a univariate analysis, OS from 
initiation of BV was significantly shorter in patients initially diagnosed with more advanced stage $\mathrm{HL}$, and significantly longer in patients who received six or more cycles of BV compared with those who received five or fewer cycles $(P<0.05$; Supplementary Table S3).

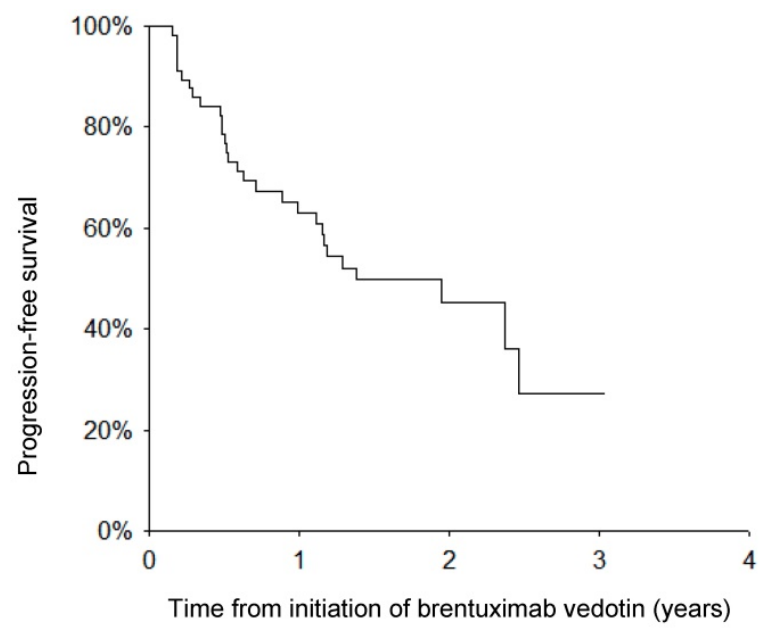

\begin{tabular}{ll}
\hline & PFS $(95 \% \mathrm{Cl})$ \\
\hline 1-year & $63.2 \%(50.2 \% ; 76.1 \%)$ \\
2-year & $45.2 \%(29.9 \% ; 60.5 \%)$ \\
\hline
\end{tabular}

$\mathrm{Cl}$, confidence interval; PFS, progression-free survival.

Figure 1: Progression-free survival of patients with Hodgkin lymphoma from initiation of brentuximab vedotin

\section{Discussion}

Our study reports and assesses data related to the treatment of $\mathrm{R} / \mathrm{R} \mathrm{HL}$, and specifically $\mathrm{BV}$, in clinical practice in a 'real-world' setting. This information can provide a valuable complement to findings from randomized clinical trials, capturing experience in routine clinical practice rather than the selected, highly controlled trial conditions.

The characteristics of the patients included in our study were typical of those encountered in real-life practice who are eligible for treatment with BV. Comparing our observational study with the pivotal phase II clinical trial of $B V$ in $R / R ~ H L$, the median age of patients (30.5 and 31 years, respectively) was the same, and the median number of prior chemotherapy regimens $(3.0$ and 3.5, respectively), proportion of patients who had previously received at least one ASCT (both 91\%) and the proportion who had received radiotherapy $(69 \%$ and $67 \%$, respectively) were all similar. 8 Some differences exist between the study populations: in the pivotal phase II trial, for example, all patients had undergone ASCT, whereas the present study included
5 patients who were ineligible for ASCT. Response assessment also differed: while the phase II trial included both computed tomography and PET scans in the trial design, the present study evaluated response by computed tomography only - PET scans were not routinely available in this retrospective analysis. While results may have been different with a PET-driven approach, this study was not a clinical trial, and PET/CT is not always a standard procedure for interim disease response evaluation in routine clinical practice, even in countries other than the Czech Republic and Slovakia.

Most patients (86\%) had received an ABVD regimen, and a substantial proportion had received BEACOPP (36\%), as part of their treatment history prior to $\mathrm{BV}$, reflecting that some patients were switched from BEACOPP to ABVD due to toxicity issues. This suggests that practice in the centers in the Czech Republic and Slovakia is consistent with ESMO guidelines, which recommend either ABVD or BEACOPP as front-line treatment for newly diagnosed HL. ${ }^{3}$ Frequently used salvage regimens included DHAP and ICE. The high proportion of patients who had previously received at least one ASCT (91\%), with the exceptions being those unsuitable for ASCT, reflects that use of BV followed the licensed indications for $\mathrm{BV}$ as well as ESMO recommendations. ${ }^{3,18}$

Seven patients $(12 \%)$ had received prior allo-SCT, consistent with ESMO guidelines that this is not a standard approach in HL but can be considered for young patients in good general condition with relapse after ASCT. ${ }^{3}$ Of these 7 patients, 4 achieved $\mathrm{CR}$ following subsequent treatment with $\mathrm{BV}$, none of whom relapsed. BV treatment in patients post-allo-SCT has thus far not been studied extensively. A previous study of 25 patients with HL relapsing after allo-SCT, indicated that BV treatment had potential utility in post-allo-SCT management, resulting in a $38 \% \mathrm{CR}$ rate. ${ }^{20}$ Although a small number, the results of the present study provide further support for the efficacy of BV treatment post-allo-SCT.

The response rates after treatment with BV are mostly consistent with previous studies. For example, the CR rate of $33 \%$ following BV treatment among patients in our clinics in the Czech Republic and Slovakia was the same as that seen in a long-term follow-up of patients in the pivotal phase II trial. ${ }^{9}$ The ORR $(47 \%)$ and CR rates in our study were also within the range reported from other observational studies (ORR: 40-73\%; CR: 18-34\%). ${ }^{12-16}$ Based on the analysis of experience in our clinics, patients who received less extensive prior treatment were more likely to achieve $\mathrm{CR}$, and subsequently a higher number of cycles of $\mathrm{BV}$ 
treatment. There was some indication that the risk of relapse was increased when the dosing interval of BV was changed from that recommended, but the small number of patients for whom relevant data were available limits interpretation. In patients with PD after BV, there was a longer interval between BV cycles. The cause of prolongation between BV cycles was most often due to infectious complications (respiratory tract infections) and myelotoxicity. It is not currently clear how to distinguish between those patients who are likely to gain the greatest benefit from BV treatment (i.e. long-term remission) and therefore do not require any further consolidation and those for whom BV therapy should serve as a bridge to allo-SCT. A recent review of published reports from the named patient program for $\mathrm{BV}$, drawing together experience from 480 patients with $R / R$ HL in

(A)

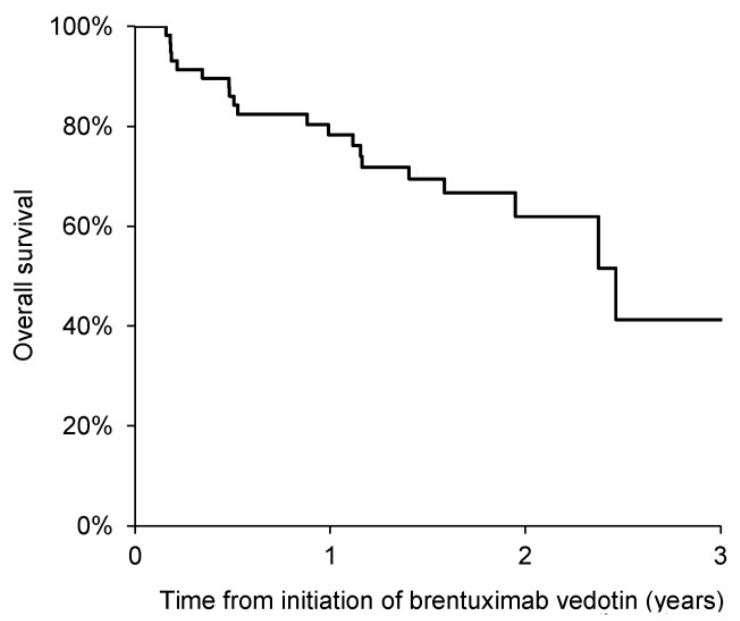

(B)

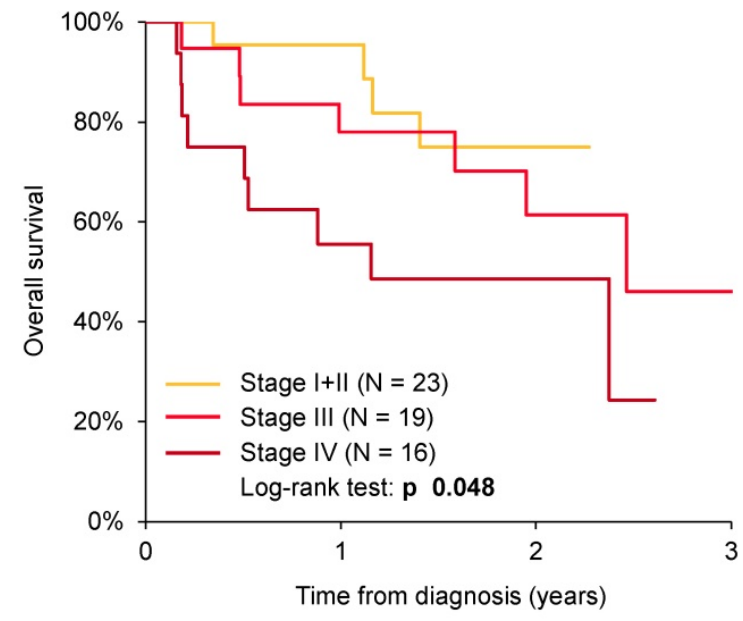

approximately 60 countries, found 1-year OS rates of $67-76 \%$ and 2 -year OS rates of $58-67 \%$ from the initiation of BV treatment. ${ }^{21}$ Survival rates in our study were consistent with the results from the named patient program, with 1- and 2-year OS rates of $78 \%$ and $62 \%$, respectively. Comparing these results in real-world settings with those from clinical trials, in the pivotal phase II trial with BV, the estimated 1-year OS rate was somewhat higher at $89 \%$ and in long-term follow-up the 5 -year OS rate was $41 \% .8,9$ The duration of therapeutic response ( 5 months) appears to be lower than that observed in previous pivotal and real-world studies.8,12,13,15,21 This may be due to the inclusion of more heavily pretreated patients with R/R HL, who had previously undergone 2-9 previous chemotherapy regimens, as well as prior ASCT and allo-SCT.

\begin{tabular}{ll}
\hline & OS (95\% Cl) \\
\hline 1-year & $78.3 \%(67.4 \% ; 89.2 \%)$ \\
2-year & $61.9 \%(46.6 \% ; 77.2 \%)$ \\
3-year & $41.3 \%(15.8 \% ; 66.8 \%)$ \\
\hline
\end{tabular}

\begin{tabular}{lccc}
\hline OS (95\% Cl) & Stage I+II & Stage III & Stage IV \\
\hline 1-year & $95.5 \%(86.8 \% ; 100.0 \%)$ & $78.0 \%(59.0 \% ; 97.1 \%)$ & $55.6 \%(30.9 \% ; 80.2 \%)$ \\
2-year & $75.0 \%(53.4 \% ; 96.6 \%)$ & $61.4 \%(36.0 \% ; 86.8 \%)$ & $48.6 \%(23.5 \% ; 73.7 \%)$ \\
3-year & - & $46.1 \%(13.8 \% ; 78.4 \%)$ & - \\
\hline
\end{tabular}

$\mathrm{CI}$, confidence interval; OS, overall survival.

Figure 2: (A) Overall survival of patients with Hodgkin lymphoma from initiation of brentuximab vedotin and (B) according to disease stage 
By drawing on real-world data, our study provides further evidence on the use and effectiveness of $B V$ in routine clinical practice. The use of $B V$ in $R / R$ HL management should be considered in the context of other treatment options, such as immune checkpoint inhibitors. ${ }^{22-25}$ Data were collected using a consistent, standardized and systematic approach that allowed a robust analysis. At the same time, common to other real-world analyses, our study is potentially open to numerous confounding factors and sources of bias that would, to some extent, be reduced by the restrictions and controls of a prospective clinical trial. The number of patients included in the analysis was also relatively small, reducing the scope for sub-analyses and interpretation, which means that valid conclusions can only be drawn from a limited number of statistical tests. In conclusion, this analysis of real-world data for patients with R/R HL in the Czech Republic and Slovakia, demonstrated efficacy with BV consistent with that seen in previous reports from real-world practice as well as clinical trials.

\section{Supplementary Material}

Supplementary tables.

http://www.jcancer.org/v10p5041s1.pdf

\section{Acknowledgments}

Medical writing support was provided by Maya Kaushik at Sciterion and Ian Faulkner at Ogilvy 4D during the preparation of this paper supported by Takeda Pharmaceuticals. Responsibility for opinions, conclusions, and interpretation of data lies with the authors.

\section{Competing Interests}

Zdeněk Král, MD, consultancy/advisory board member: Takeda, speaker for: Amgen, Teva, research support from: no, stock options with: no, consultancy for: Takeda.

Jozef Michalka, MD, consultancy/advisory board member: no, speaker for: no, research support from: no, stock options with: no, consultancy for: no.

Heidi Móciková, MD, consultancy/advisory board member: no, speaker for: no, research support from: no, stock options with: no, consultancy for: no.

Jana Marková, MD, consultancy/advisory board member: no, speaker for: no, research support from: no, stock options with: no, consultancy for: no.

Alice Sýkorová, MD, consultancy/advisory board member: no, speaker for: Bristol-Myers Squibb, Roche, Takeda research support from: no, stock options with: no, consultancy for: no.

David Belada, MD, consultancy/advisory board member: Takeda, speaker for: Takeda, research support from: Takeda, stock options with: no, consultancy for: no.

Alexandra Jungová, MD, consultancy/advisory board member: no, speaker for: no, research support from: no, stock options with: no, consultancy for: no.

Samuel Vokurka, MD, consultancy/advisory board member: Takeda, speaker for: no, research support from: no, stock options with: no, consultancy for: Novartis, Roche, Teva.

Marie Lukášová, MD, consultancy/advisory board member: no, speaker for: Bristol-Myers Squibb, research support from: no, stock options with: no, consultancy for: no.

Vít Procházka, MD, consultancy/advisory board member: Bristol-Myers Squibb, Takeda, speaker for: Gilead, Servier, research support from: Takeda, stock options with: no, consultancy for: no.

Juraj Ďuraš, MD, consultancy/advisory board member: Roche, speaker for: no, research support from: no, stock options with: no, consultancy for: no.

Roman Hájek, MD, consultancy/advisory board member: Amgen, Bristol-Myers Squibb, Celgene, Janssen-Cilag, Takeda, speaker for: no, research support from: Janssen-Cilag, Takeda, stock options with: no, consultancy for: Amgen, Bristol-Myers Squibb, Celgene, Janssen-Cilag, Takeda, honoraria from: Amgen, Bristol-Myers Squibb, Celgene, Janssen-Cilag and Takeda.

Ladislav Dušek MD, consultancy/advisory board member: no; speaker for: no, research support from: no, stock options with: no, consultancy for: no.

Luboš Drgoňa MD, consultancy/advisory board member: AbbVie, Bristol-Myers Squibb, Celgene, Gilead, JnJ/Janssen, Pfizer, Roche/Genentech, Servier, Takeda; speaker for: Celgene, Pfizer, Roche/Genentech, Servier, Teva, research support from: no, stock options with: no, consultancy for: no.

Miriam Ladická MD, consultancy/advisory board member Celgene, Roche/Genentech, Servier, Takeda, speaker for: Celgene, Roche/Genentech, Servier, Takeda, research support from: no, stock options with: no, consultancy for: no.

Veronika Ballová MD, consultancy/advisory board member -no; speaker for: -no, research support from: -no, stock options with: no, consultancy for: no.

Andrej Vranovský MD, consultancy/advisory board member of: Celgene, Gilead, Janssen, Roche, Takeda, speaker for: Roche, research support from: Celgene, stock options with: no, consultancy for: no.

\section{References}

1. Bonadonna $G$, Bonfante V, Viviani S, et al. ABVD plus subtotal nodal versus involved-field radiotherapy in early-stage Hodgkin's disease: long-term results. J Clin Oncol. 2004; 22: 2835-41.

2. Engert A, Diehl V, Franklin J, et al. Escalated-dose BEACOPP in the treatment of patients with advanced-stage Hodgkin's lymphoma: 10 years of follow-up of the GHSG HD9 study. J Clin Oncol. 2009; 27: 4548-54. 
3. Eichenauer DA, Alemen BMP, André M, et al. Hodgkin lymphoma: ESMO Clinical Practice Guidelines for diagnosis, treatment and follow-up. Ann Oncol. 2018; 29(suppl 4): iv19-29.

4. Bhatt VR, Loberiza FR Jr, Jing H, et al. Mortality patterns among recipients of autologous hematopoietic stem cell transplantation for lymphoma and myeloma in the past three decades. Clin Lymphoma Myeloma Leuk. 2015; 15: 409-15.

5. Moskowitz C, Kewalramani T, Nimer SD, et al. Risk-adapted high dose chemoradiotherapy and ASCT for patients with relapsed or refractory Hodgkin's disease: An intent to treat analysis. Blood. 2003; 102: 118a. Abstract 403.

6. Moskowitz AJ, Perales MA, Kewalramani T, et al. Outcomes for patients who fail high dose chemoradiotherapy and autologous stem cell rescue for relapsed and primary refractory Hodgkin lymphoma. Br J Haematol. 2009; 146: 158-63.

7. Martínez C, Canals C, Sarina B, et al. Identification of prognostic factors predicting outcome in Hodgkin's lymphoma patients relapsing after autologous stem cell transplantation. Ann Oncol. 2013; 24: 2430-4.

8. Younes A, Gopal AK, Smith SE, et al. Results of a pivotal phase II study of brentuximab vedotin for patients with relapsed or refractory Hodgkin's lymphoma. J Clin Oncol. 2012; 30: 2183-9.

9. Chen R, Gopal AK, Smith SE, et al. Five-year survival and durability results of brentuximab vedotin in patients with relapsed or refractory Hodgkin lymphoma. Blood.2016; 128: 1562-6.

10. Forero-Torres A, Fanale $M$, Advani $R$, et al. Brentuximab vedotin in transplant-naive patients with relapsed or refractory Hodgkin lymphoma: analysis of two phase I studies. Oncologist. 2012; 17: 1073-80.

11. Viviani S, Guidetti A, Dalto S, et al. Brentuximab vedotin (BV) an effective treatment for transplant ineligible patients with relapsed/refractory (R/R) Hodgkin lymphoma (HL). Blood. 2014; 124: abstract E1142.

12. Monjanel H, Deville L, Ram-Wolff C, et al. Brentuximab vedotin in heavily treated Hodgkin and anaplastic large-cell lymphoma, a single centre study on 45 patients. Br J Haematol. 2014; 166: 306-8.

13. Salihoglu A, Elverdi T, Karadogan I, et al. Brentuximab vedotin for relapsed or refractory Hodgkin lymphoma: experience in Turkey. Ann Hematol. 2015; 94: $415-20$.

14. Yang QM, Hong JY, Ko YH, et al. Brentuximab vedotin for relapsed or refractory CD30+ Hodgkin lymphoma: a multicenter analysis from Asia. Onco Targets Ther. 2014; 7: 1717-22.

15. Zinzani PL, Viviani S, Anastasia A, et al. Brentuximab vedotin in relapsed/refractory Hodgkin's lymphoma: the Italian experience and results of its use in daily clinical practice outside clinical trials. Haematologica. 2013; 98: 1232-6.

16. Zinzani PL, Pellegrini C, Cantonetti $M$, et al. Brentuximab vedotin in transplant-naïve relapsed/refractory Hodgkin lymphoma: experience in 30 patients. Oncologist. 2015; 20: 1413-6.

17. Cheson BD, Pfistner B, Juweid ME, et al. Revised response criteria for malignant lymphoma. J Clin Oncol. 2007; 25: 579-86.

18. Adcetris $®$. Summary of Product Characteristics. January 2018.

19. Moskowitz $\mathrm{CH}$, Nademanee A, Masszi T, et al. Brentuximab vedotin as consolidation therapy after autologous stem-cell transplantation in patients with Hodgkin's lymphoma at risk of relapse or progression (AETHERA): a randomised, double-blind, placebo-controlled, phase 3 trial. Lancet. 2015; 385: 1853-61.

20. Gopal AK, Ramchandren R, O'Connor OA, et al. Safety and efficacy of brentuximab vedotin for Hodgkin lymphoma recurring after allogeneic stem cell transplantation. Blood. 2012; 120: 560-8.

21. Zinzani PL, Sasse S, Radford J, et al. Brentuximab vedotin in relapsed/refractory Hodgkin lymphoma: An updated review of published data from the named patient program. Crit Rev Oncol Hematol. 2016; 104: 65-70.

22. Armand P, Engert A, Younes A, et al. Nivolumab for relapsed/refractory classic Hodgkin lymphoma after failure of autologous hematopoietic cell transplantation: Extended follow-up of the multicohort single-arm Phase II CheckMate 205 trial. J Clin Oncol. 2018; 36: 1428-39.

23. Chen R, Zinzani PL, Fanale MA, et al. Phase II study of the efficacy and safety of pembrolizumab for relapsed/refractory classic Hodgkin lymphoma. J Clin Oncol. 2017; 35: 2125-32.

24. Herrera AF, Moskowitz AJ, Bartlett NL, et al. Results from a Phase 1/2 study of brentuximab vedotin in combination with nivolumab in patients with relapsed or refractory Hodgkin lymphoma. Presented at the 59th Annual Meeting of the American Society of Hematology. 2017; Abstract 649.

25. Armand P, Shipp MA, Ribrag V, et al. Programmed death-1 blockade with pembrolizumab in patients with classical Hodgkin lymphoma after brentuximab vedotin failure. J Clin Oncol. 2016; 34: 3733-9. 